\title{
Unusual case of locally advanced and metastatic paratesticular liposarcoma: A case report and review of the literature
}

\author{
Stefano Chiodini ${ }^{1}$, Lorenzo G. Luciani ${ }^{1}$, Tommaso Cai ${ }^{1}$, Alberto Molinari ${ }^{2}$, Luca Morelli ${ }^{3}$, \\ Chiara Cantaloni $^{3}$, Mattia Barbareschi ${ }^{3}$, Gianni Malossini ${ }^{1}$ \\ ${ }^{1}$ Department of Urology, Santa Chiara Hospital, Trento, Italy; \\ ${ }^{2}$ Department of Urology, Don Calabria-Sacro Cuore Hospital, Negrar (VR), Italy; \\ ${ }^{3}$ Department of Pathology, Santa Chiara Hospital, Trento, Italy.
}

\begin{abstract}
Summary Liposarcoma accounts for $20 \%$ of all sarcomas and is a rare occurrence in the paratesticular region.

We present the case of a 66-year-old man with a massive liposarcoma of the right scrotum invading the lower limb and the abdominal wall skin. The case concerns an unusually large and aggressive liposarcoma $(25 \mathrm{~cm})$, presenting with multiple lung and nodal metastases. The patient had an unfavourable evolution with rapid progression of metastases, although there were no signs of local disease. In this case, a wide local excision was performed in order to obtain local control of the disease. Even though paratesticular sarcomas might have a more favourable evolution, the association with lung involvement carries an ominous prognosis. Diagnosis of paratesticular sarcoma should be kept in mind in case of irregular necrotic mass$e s$ in the inguinal and scrotal region.
\end{abstract}

KEY WORDS: Liposarcoma; Testicular neoplasms; Case report; Spermatic cord; Orchiectomy.

Submitted 15 February 2015; Accepted 2 March 2015

\section{INTRODUCTION}

Liposarcoma accounts for $20 \%$ of all sarcomas and is a rare occurrence in the paratesticular region.

Approximately 200 cases of paratesticular liposarcoma (PLS) have been reported in the literature (1).

The optimal treatment of these tumours remains controversial, but there is a general consensus that all paratesticular sarcomas in adults should be managed with complete resection, including high ligation of the spermatic cord (2-5). We report one of the largest PLS with an unfavourable prognosis in spite of aggressive surgical treatment. We present the case of a 66-year-old man with a massive liposarcoma of the right scrotum invading the lower limb and the abdominal wall skin.

\section{Case report}

A 66-year-old man was referred for a bulky painless mass of the right inguinal and scrotal region associated with necrotic ulceration of the skin. General conditions at presentation were poor; the patient reported a slowly growing mass since one year.

Medical history included previous cholecystectomy, hypertension and mild lower urinary tract symptoms. Physical examination revealed a large solid mass extending from the right scrotum to the inguinal region and to the ipsilateral thigh, associated with a deep necrotic ulceration at the root of the scrotum. The right testis was increased in volume and hard. The left testis and the penis were intact and dislodged laterally by the mass. Beta-Human Chorionic Gonadotropin, alpha-fetoprotein, and lactate dehydrogenase were normal, while Creactive Protein and leukocytes were elevated. Staging CT scan showed a $25 \mathrm{~cm}$ paratesticular solid mass with colliquative areas, slightly increased nodes in the inguinal and external iliac region, and multiple pulmonary solid nodules ranging from a few $\mathrm{mm}$ to $3 \mathrm{~cm}$. The patient underwent surgical wide excision of the mass, including the right testicle and all right inguinal canal contents up to the deep inguinal ring by inguinotomy access (inguinal incision). No postoperative complications were reported. Histopathology revealed an undifferentiated liposarcoma infiltrating the testis abscessed to the overlying skin with extensive necrotic areas. Adjuvant chemotherapy, initially suggested, was afterwards aborted due to cardiological complications and supportive care only was provided. At follow-up CT scan at 3 and 6 months, progression of lung metastases and right inguinal-external iliac lymph nodes was reported; surgical wound was regular. The patient died 9 months postoperatively for respiratory insufficiency due to further progression of lung metastases.

\section{Discussion}

Sarcomas of the genitourinary tract account for fewer than $5 \%$ of sarcomas. The first case of a spermatic cord sarcoma was reported in 1845 by Lesauvage (6).

Sarcomas of soft tissue origin derive embryologically from mesodermal tissues and can occur in many organs. 


\section{Figure 1.}

The figure shows large solid mass with deep necrotic ulceration at the root of the right scrotum.

Panel A: patient at presentation.

Panel B: surgical specimen.
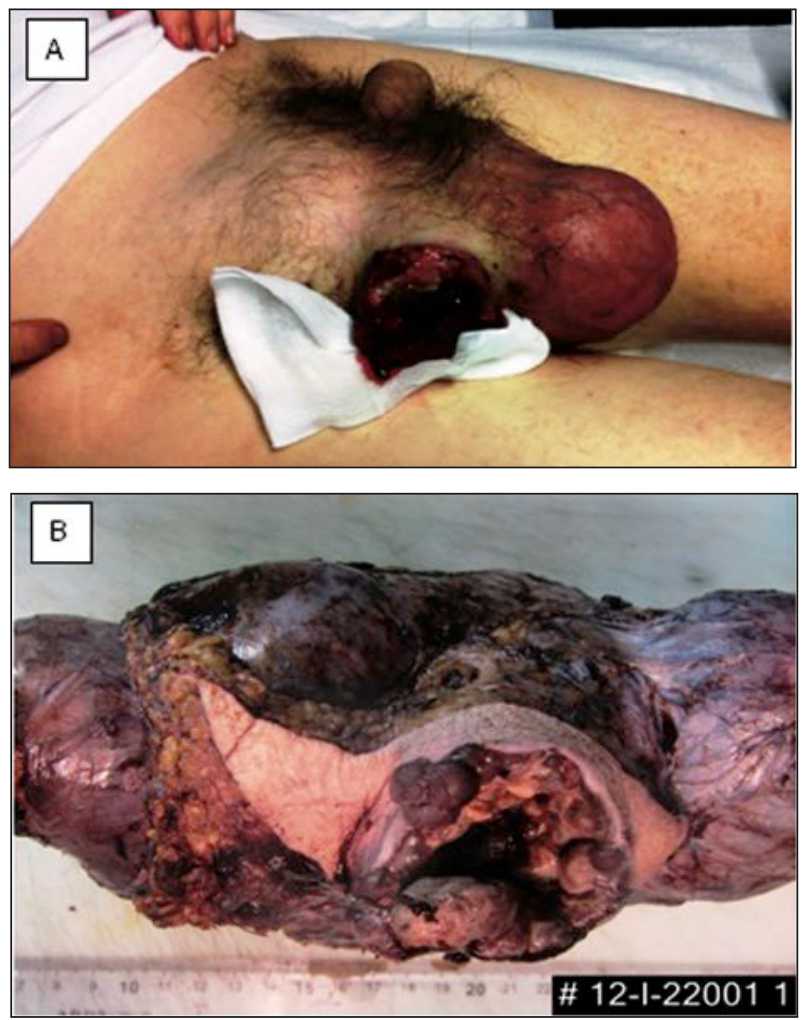

Figure 2.

The figure shows microscopic findings.

Panel A: Area of dedifferentiated liposarcoma. Presence of large, round cells with nuclear abnormalities in a background of spindle and rounded cells (EE, 20x).

Panel B: Particular of previous picture (EE, 40x).
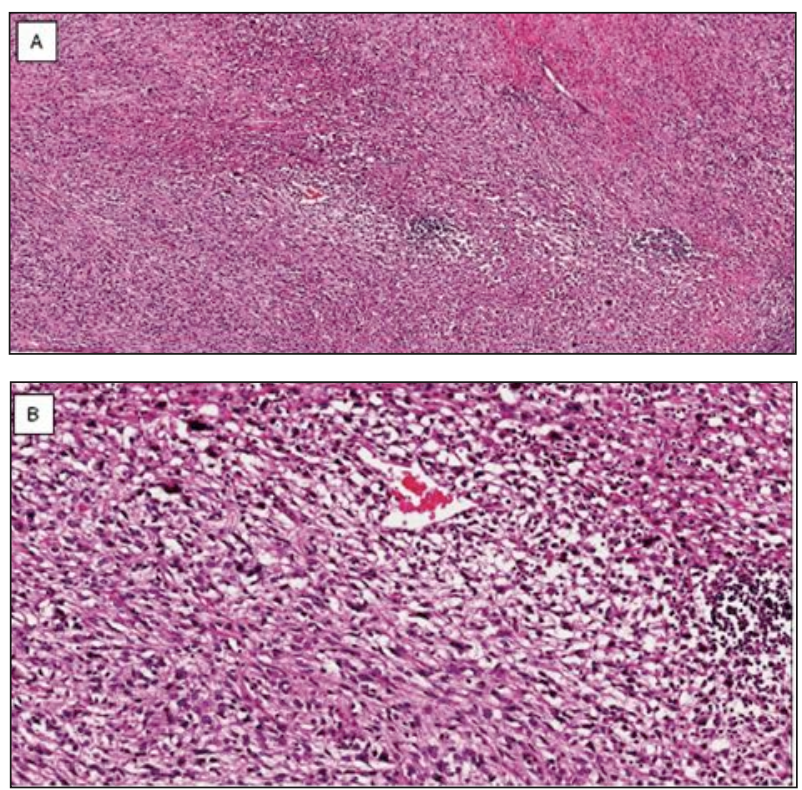

Although rare in the genitourinary tract, they can occur in spermatic cord, scrotum, and epididymis and resemble their counterparts in other organs. In adults, liposarcomas represent the most common soft-tissue sarcomas, and usually occur in patients older than 40 years. Liposarcomas of the spermatic cord usually begin to grow directly below the external inguinal ring, so when the tumours reach a large size, they present as painless scrotal rather than inguinal mass (7). PLS most commonly originates in the spermatic cord; however, because of the difficulty in determining the exact site of origin, PLS include all liposarcomas rising in the structures around the testis, including the lower end of the cord. These tumours are often well-differentiated, and only $40 \%$ of the poorly differentiated liposarcomas metastasize (8). The diagnosis is usually obtained by ultrasound detection of an indolent scrotal or inguinal mass. Additional radiologic studies might detect retroperitoneal or pulmonary involvement. Sarcomas tend to infiltrate local tissues widely, making complete resection difficult. It has been suggested that at least a third of patients after local excision or orchidectomy occult local residual disease (4). Therefore, primary treatment should consist of a wide local excision, orchiectomy and with re-excision, if needed, to ensure negative margins. Retroperitoneal lymphadenectomy is used for some cases of rhabdomyosarcoma and for other sarcomas with proven lymphatic invasion. Mortality and morbidity depend on histologic tumour type, tumour stage, and the presence of sarcoma at the surgical margin of the specimen. Well-differentiated liposarcomas tend to recur over many years, but they do not metastasize. There are no large studies of liposarcomas with adequate follow-up, but myxoid/round cell and de-differentiated liposarcomas may persist, recur, or metastasize (7).

Recurrences are seen in $1 / 4$ patients and metastases are seen in 1/10 patients with spermatic cord liposarcoma through lymphatic or haematogenous spread. If a sufficient tumour-free margin cannot be achieved, adjuvant radiotherapy covering the inguinal region and scrotum should be considered. The case we report concerns an unusually large and aggressive liposarcoma $(25 \mathrm{~cm})$, presenting with multiple lung and nodal metastases. The patient had an unfavourable evolution with rapid progression of metastases, although there were no signs of local disease. In this case, a wide local excision was performed in order to obtain local control of the disease. Even though paratesticular sarcomas might have a more favourable evolution, the association with lung involvement carries an ominous prognosis. Diagnosis of paratesticular sarcoma should be kept in mind in case of irregular necrotic masses in the inguinal and scrotal region.

\section{Conclusion}

The relative paucity of cases reported in the literature makes it difficult to document the natural history for such a tumour. The information used to guide treatment is based on previous experiences from groups of few patients: no clear consensus exists regarding the proper treatment. Radical orchiectomy with wide local excision provides ideally the best chance of eradicating this dis- 
ease; however, the association with distant metastases implies an ominous prognosis. The diagnosis of liposarcoma should be kept in mind in case of a large solid mass in the paratesticular region with irregular margins.

\section{REFERENCES}

1. Frank I, Takahashi S, Tsukamoto T, Lieber MM. Genitourinary sarcomas and carcinosarcomas in adults. In: Vogelzang NJ, Shipley WU, Scardino PT, Coffey DS, eds. Comprehensive Textbook of Genitourinary Oncology. $2^{\text {nd }} \mathrm{ed}$. Philadelphia: Lippincott Williams E Wilkins. 2000; 1110-3.

2. Merimsky O, Terrier P, Bonvalot $S$, et al. Spermatic cord sarcoma in adults. Acta Oncologica. 1999; 38:35-8.

3. Catton CN, Cummings V, Fornasier B, et al. Adult paratesticular sarcomas. A review of 21 cases. J Urol. 1991; 146:342-5.
4. Catton CN, Jewett M, O'Sullivan I, Kandel R. Paratesticular sarcoma: Failure pattern after definitive local therapy. J Urol. 1999; 161:1844-7.

5. Fagundes MA, Zietman AL, Althausen AF, et al. The management of spermatic cord sarcoma. Cancer. 1996; 77:1873-6.

6. F. Hinman F, Gibson TE. Tumors of the epididymis, spermatic cord and testicular tunics: a review of literature and report of three new cases. Archives of Surgery 1824; 8: article 100.

7. Zhou M, Magi-Galluzzi C. Genitourinary Pathology: A Volume in the Series Foundations in Diagnostic Pathology. First Edition. Chapter 10, pp.534-622.

8. Stout AP. Liposarcoma - the malignant tumor of lipoblasts. Ann Surg. 1944; 119:86-107.

\section{Correspondence}

Stefano Chiodini, MD (Corresponding Author)

stefano.chiodini@apss.tn.it

Lorenzo G. Luciani, MD

Tommaso Cai, MD

Department of Urology, Santa Chiara Hospital

Largo Medaglie d'oro 9, 38122 Trento, Italy

Alberto Molinari, MD

Department of Urology, Don Calabria-Sacro Cuore Hospital, Negrar (VR), Italy

Luca Morelli, MD

Chiara Cantaloni, MD

Mattia Barbareschi, MD

Gianni Malossini, MD

Department of Urology, Santa Chiara Hospital, Trento, Italy 